\title{
Clinical Impact of Stomach-partitioning Gastrojejunostomy with Braun Enteroenterostomy for Patients with Gastric Outlet Obstruction Caused by Unresectable Gastric Cancer
}

\author{
TAKAAKI ARIGAMI ${ }^{1,2}$, YOSHIKAZU UENOSONO $^{2}$, SUMIYA ISHIGAMI ${ }^{1}$, \\ SHIGEHIRO YANAGITA ${ }^{1}$, KEISHI OKUBO ${ }^{1}$, YASUTO UCHIKADO ${ }^{1}$, YOSHIAKI KITA $^{1}$, \\ SHINICHIRO MORI ${ }^{1}$, HIROSHI KURAHARA ${ }^{1}$, KOSEI MAEMURA ${ }^{1}$ and SHOJI NATSUGOE ${ }^{1,2}$ \\ ${ }^{1}$ Department of Digestive Surgery, Breast and Thyroid Surgery, \\ Kagoshima University Graduate School of Medical and Dental Sciences, Kagoshima, Japan; \\ ${ }^{2}$ Molecular Frontier Surgery, Kagoshima University Graduate School of Medical and Dental Sciences, Kagoshima, Japan
}

\begin{abstract}
Aim: To compare adverse events and posttherapeutic clinical courses between stomach-partitioning gastrojejunostomy with Braun enteroenterostomy (SPGJ-BEE) and endoscopic metallic stent placement (EMSP) in patients with gastric outlet obstruction (GOO) caused by unresectable gastric cancer and assess the clinical utility of SPGJ-BEE. Patients and Methods: We retrospectively reviewed clinical data of 16 and 9 patients with GOO undergoing SPGJ-BEE and EMSP, respectively. Results: Re-obstruction caused by tumor overgrowth was identified in $3(33.3 \%)$ out of 9 patients in the EMSP group. The GOO scoring system (GOOSS) revealed that its score after treatments was significantly higher in the SPGJ-BEE group than in the EMSP group $(p<0.001)$. All patients in both groups received chemotherapy after treatments. The median survival times in the SPGJ-BEE and EMSP groups were 414 and 303 days, respectively. Conclusion: Our preliminary results suggest that SPGJ-BEE provides an improved long-term quality of life and the early induction of subsequent chemotherapy related with a better prognosis in patients with GOO.
\end{abstract}

Gastric cancer is one of the most common gastrointestinal tract malignancies in Asia, including Japan $(1,2)$. The

Correspondence to: Takaaki Arigami, MD, Ph.D., Department of Digestive Surgery, Breast and Thyroid Surgery, and Molecular Frontier Surgery, Kagoshima University Graduate School of Medical and Dental Sciences, 8-35-1 Sakuragaoka, Kagoshima 8908520, Japan. Tel: +81 992755361, Fax: +81 992657426, e-mail: arigami@m.kufm.kagoshima-u.ac.jp

Key Words: Gastric outlet obstruction, stomach-partitioning gastrojejunostomy, Braun enteroenterostomy, endoscopic metallic stent placement, gastric cancer. incidence of gastric outlet obstruction (GOO) ranges between $14.9 \%$ and $35.0 \%$ in patients with antral gastric cancer (3, 4). Endoscopic metallic stent placement (EMSP) has been widely accepted as an endoscopic treatment for functional disorders caused by GOO (5). On the other hand, gastrojejunostomy (GJ) is one of the surgical palliative treatments available for patients with GOO caused by unresectable tumors, such as gastric cancer, duodenal cancer and pancreatic cancer. Although GJ had conventionally been performed using hand-sewn sutures, circular or linear stapled GJ has become more common due to recent advances in surgical apparatuses and an increase in laparoscopic surgical approaches (6). Furthermore, GJ, using these staplers, may produce convenient anastomosis in laparoscopic surgery (6).

Delayed gastric emptying (DGE) is one of the postoperative complications that occur in patients after GJ and its incidence has been reported to be between $10 \%$ and $26 \%(7,8)$. Therefore, novel surgical methods have been developed in order to avoid DGE after GJ. Several investigators have demonstrated the clinical utility of stomach-partitioning gastrojejunostomy (SPGJ) in patients with GOO $(6,9-11)$. They demonstrated that SPGJ is an effective surgical approach to prevent DGE and tumor bleeding by food contact (9-11). Additionally, it allows for tumor conditions to be observed via the drainage route on the lesser curvature after SPGJ. Moreover, recent studies have focused on the clinical benefits of Braun enteroenterostomy (BEE) in several surgical procedures $(12,13)$. Nevertheless, it is important to preserve postoperative good conditions in the clinical management of patients with GOO after GJ.

To date, we have actively performed stomach-partitioning gastrojejunostomy with Braun enteroenterostomy (SPGJBEE), based on the surgical advantages of SPGJ and BEE, in patients with GOO. Since the surgical resection rate in patients with GOO is slightly lower than that in patients 
without GOO, the early induction of chemotherapy is a key issue for improving prognoses in patients with GOO (14). Consequently, its actualization depends on postoperative clinical conditions after SPGJ-BEE in patients with GOO. However, the clinical impact of SPGJ-BEE in patients with GOO caused by unresectable gastric cancer has not yet been established. Therefore, the aim of the present study was to compare adverse events and postoperative clinical courses between SPGJ-BEE and EMSP in patients with GOO caused by unresectable gastric cancer and assess the clinical utility of SPGJ-BEE in patients with GOO.

\section{Patients and Methods}

Patients. We retrospectively reviewed clinical data of 25 patients with GOO caused by unresectable gastric cancer who underwent SPGJBEE or EMSP as an initial palliative treatment at Kagoshima University Hospital, Japan, between May 2007 and August 2015. All patients were assessed by esophagogastroduodenoscopy, fluoroscopy, endoscopic ultrasonography and computed tomography before SPGJBEE or EMSP. All patients were then pathologically confirmed to have adenocarcinoma of the stomach by endoscopic biopsy and clinically diagnosed with unresectable gastric cancer with GOO before treatments. Twenty-three patients had primary gastric tumors with distant metastasis, while two had locally advanced gastric tumors with the involvement of the pancreas. Six out of the 23 patients with distant metastasis had 2 distant metastatic sites. In the present study, GOO was defined as a condition inducing any dyspeptic symptoms associated with significant body weight loss and malnutrition, such as nausea, early satiety and vomiting. Patients were classified and staged based on the tumor-node-metastasis classification for gastric carcinoma established by the Union for International Cancer Control (15). Survival duration was determined from the date of SPGJ-BEE or EMSP to the date of death or last follow-up. The median followup period was 340 days (range $=125-1,670$ ).

This retrospective observational and historical cohort study was approved by the Ethics Committee of Kagoshima University.

Procedures of stomach-partitioning gastrojejunostomy with BEE and EMSP. The SPGJ-BEE procedure was conducted as follows: stomach-partitioning was performed using a linear stapler (EndoGIA Universal $^{\mathrm{TM}}$ 60-3.5; Covidien, Mansfield, MA, USA) on an approximately $3-\mathrm{cm}$ proximal part from the oral side of tumors at the body of the stomach after mobilizing the omentum and gastroepiploic vascular arcade (Figure 1). The stomach was then transected from the greater curvature side and an approximately 2.0$\mathrm{cm}$ residual lumen on the lesser curvature side was retained to permit endoscopic tumor surveillance. The partitioned proximal part of the stomach was inosculated to the jejunum, approximately $30 \mathrm{~cm}$ from the ligament of Treitz, using a linear stapler (EndoGIA Universal ${ }^{\mathrm{TM}}$ 60-3.5) as side-to-side anastomosis (Figure 1). Antecolic GJ was performed on all patients in the SPGJ-BEE group. BEE was created $10 \mathrm{~cm}$ distal from the GJ using a linear stapler (EndoGIA Universal $^{\mathrm{TM}}$ 60-2.5) as side-to-side anastomosis (Figure 1).

EMSP was performed using an Enteral Wallflex stent (Boston Scientific, Natick, MA, USA) with a diameter of $22 \mathrm{~mm}$ and length of 60 or $90 \mathrm{~mm}$. The metallic stent was inserted over a guidewire (Jagwire; Boston Scientific) and deployed under endoscopic and fluoroscopic monitoring.
Table I. Clinical characteristics of patients with gastric outlet obstruction caused by unresectable gastric cancer.

\begin{tabular}{|c|c|c|c|}
\hline Factor & $\begin{array}{l}\text { SPGJ-BEE }(\%) \\
\quad(n=16)\end{array}$ & $\begin{array}{l}\operatorname{EMSP}(\%) \\
\quad(\mathrm{n}=9)\end{array}$ & $p$-Value \\
\hline \multicolumn{4}{|l|}{ Gender } \\
\hline Male & $11(68.8)$ & $4(44.4)$ & 0.397 \\
\hline Female & $5(31.2)$ & $5(55.6)$ & \\
\hline Age (mean years) & $66.6 \pm 13.2$ & $76.0 \pm 8.2$ & 0.047 \\
\hline \multicolumn{4}{|l|}{ ECOG performance status } \\
\hline $0-1$ & $16(100.0)$ & $3(33.3)$ & $<0.001$ \\
\hline 2 & $0(0.0)$ & $6(66.7)$ & \\
\hline \multicolumn{4}{|l|}{ Macroscopic type } \\
\hline Type 4 & $3(18.8)$ & $5(55.6)$ & 0.087 \\
\hline Non-type 4 & $13(81.2)$ & $4(44.4)$ & \\
\hline \multicolumn{4}{|l|}{ Tumor location } \\
\hline Middle & $1(6.2)$ & $8(88.9)$ & $<0.001$ \\
\hline Lower & $15(93.8)$ & $1(11.1)$ & \\
\hline \multicolumn{4}{|l|}{ Depth of tumor invasion } \\
\hline cT3 & $1(6.2)$ & $0(0.0)$ & 1.000 \\
\hline cT4 & $15(93.8)$ & $9(100.0)$ & \\
\hline \multicolumn{4}{|l|}{ Lymph node metastasis } \\
\hline cNO & $1(6.2)$ & $2(22.2)$ & 0.530 \\
\hline $\mathrm{cN} 1-\mathrm{N} 3$ & $15(93.8)$ & $7(77.8)$ & \\
\hline \multicolumn{4}{|l|}{ Distant metastasis } \\
\hline M0 & $2(12.5)$ & $0(0.0)$ & 0.520 \\
\hline M1 & $14(87.5)$ & $9(100.0)$ & \\
\hline \multicolumn{4}{|l|}{ Clinical stage } \\
\hline III & $2(12.5)$ & $0(0.0)$ & 0.520 \\
\hline IV & $14(87.5)$ & $9(100.0)$ & \\
\hline BMI, mean & $22.7 \pm 3.7$ & $21.2 \pm 4.3$ & 0.235 \\
\hline Albumin, mean (g/dl) & $3.6 \pm 0.7$ & $3.3 \pm 0.3$ & 0.156 \\
\hline CEA, mean (ng/ml) & $12.0 \pm 26.9$ & $6.5 \pm 9.1$ & 0.713 \\
\hline CA $19-9$, mean $(\mathrm{U} / \mathrm{ml})$ & $846.4 \pm 2288.7$ & $177.7 \pm 397.4$ & 0.336 \\
\hline
\end{tabular}

SPGJ-BEE, Stomach-partitioning gastrojejunostomy with Braun enteroenterostomy; EMSP, endoscopic metallic stent placement; ECOG, Eastern Cooperative Oncology Group; BMI, body mass index.

The GOO scoring system and delayed gastric emptying. Oral intake was evaluated by the gastric outlet obstruction scoring system (GOOSS) proposed by Adler and Baron: 0, no oral intake; 1, liquid only; 2, soft solid food; and 3, a low-residue or full diet (16). Posttherapeutic GOOSS scores were assessed approximately 2 weeks after SPGJ-BEE or EMSP.

DGE was defined by the International Study Group of Pancreatic Surgery (ISGPS) criteria (17). These criteria were determined based on the duration of nasogastric intubation or the reinsertion of a nasogastric tube, the period of inability to tolerate a solid diet, the presence or absence of vomiting and gastric distension and the use of prokinetic agents (17).

Statistical analysis. Differences in categorical clinicopathological factors, adverse events and GOOSS scores between the SPGJ-BEE and EMSP groups were assessed using the Chi-squared and Fisher's exact tests. Differences in age, body mass index (BMI), albumin, carcinoembryonic antigen (CEA), carbohydrate antigen 19-9 (CA199), time to a liquid or solid diet and time to the initiation of chemotherapy between the SPGJ-BEE and EMSP groups were evaluated using the Wilcoxon rank sum test. Survival curves were 


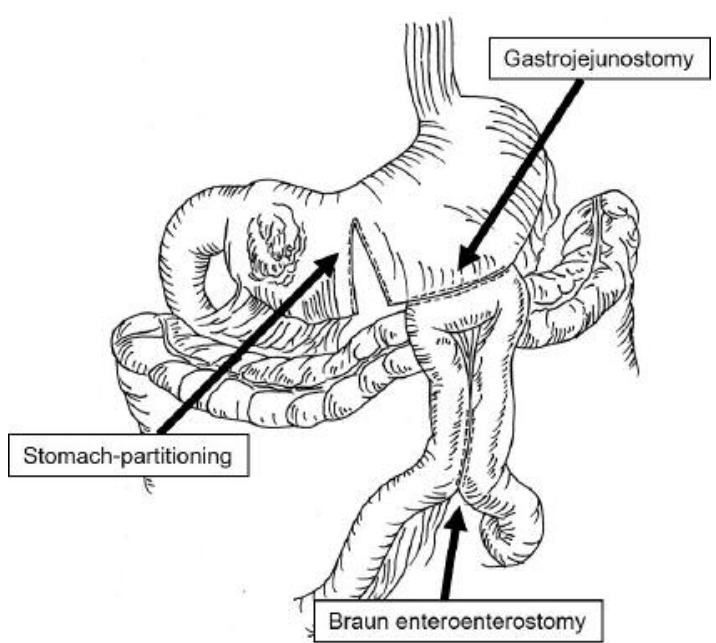

Figure 1. Schematic diagram of stomach-partitioning gastrojejunostomy with Braun enteroenterostomy.

constructed using the Kaplan-Meier method and prognostic differences were determined using the log-rank test. All data were statistically analyzed using SAS statistical software (SAS Institute Inc., Cary, NC, USA). A $p$-value of $<0.05$ was considered significant.

\section{Results}

Patients' characteristics. Twenty-five patients with GOO caused by unresectable gastric cancer were enrolled in this study. According to the initial treatment for GOO, all patients were divided into groups with SPGJ-BEE and EMSP. Table I shows clinicopathological factors in the SPGJ-BEE and EMSP groups. Sixteen patients underwent SPGJ-BEE, while 9 underwent EMSP. The mean ages $( \pm$ SD) of the SPGJ-BEE and EMSP groups were $66.6 \pm 13.2$ and $76.0 \pm 8.2$ years, respectively. The SPGJ-BEE group was significantly younger than the EMSP group $(p=0.047)$. All patients in the SPGJ-BEE group had an Eastern Cooperative Oncology Group (ECOG) performance status (PS) of 0-1. In the EMSP group, 3 and 6 patients had ECOG PS of 0-1 and PS of 2, respectively. The SPGJ-BEE group had significantly better PS than the EMSP group $(p<0.001)$. Moreover, the incidence of tumors located on the lower portion of the stomach was significantly higher in the SPGJ-BEE group than in the EMSP group $(p<0.001)$. However, no significant differences were observed in other categorical clinicopathological factors, such as gender, macroscopic type, clinical stage, BMI, albumin, CEA or CA 19-9 between the SPGJ-BEE and EMSP groups.

Adverse events. Only one patient (6.3\%) developed a surgical site infection in the SPGJ-BEE group (Table II). However,

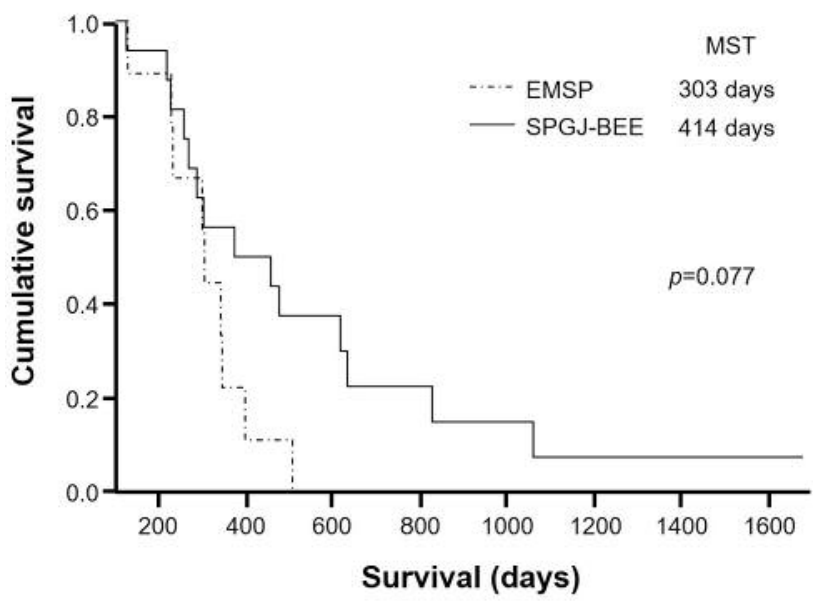

Figure 2. Kaplan-Meier survival curves after stomach-partitioning gastrojejunostomy with Braun enteroenterostomy (SPGJ-BEE) and endoscopic metallic stent placement (EMSP). The median survival times (MST) in the SPGJ-BEE and EMSP groups were 414 and 303 days, respectively.

none of the patients in the SPGJ-BEE group had anastomotic leakage, DGE or re-obstruction of anastomosis. On the other hand, bleeding, pneumonia and DGE (grade A) occurred in one patient each (11.1\%) in the EMSP group. Re-obstruction caused by tumor overgrowth was identified in $3(33.3 \%)$ out of 9 patients in the EMSP group (Table II). Therefore, reinterventions were performed on 2 patients $(22.2 \%)$ in the EMSP group. The incidence of re-obstruction was significantly higher in the EMSP group than in the SPGJBEE group $(p=0.037)$. Treatment-related death was not identified in either group.

Clinical courses. The mean times $( \pm \mathrm{SD})$ from treatments to a liquid diet in the SPGJ-BEE and EMSP groups were $3.4 \pm 2.0$ and $0.9 \pm 0.6$ days, respectively (Table III). Patients in the EMSP group had a significantly shorter time to a liquid diet than those in the SPGJ-BEE group $(p<0.001)$. The mean times $( \pm \mathrm{SD})$ from treatments to a solid diet in the SPGJ-BEE and EMSP groups were $4.8 \pm 1.9$ and $2.4 \pm 1.6$ days, respectively (Table III). Accordingly, the mean time to a solid diet was significantly shorter in the EMSP group than in the SPGJ-BEE group $(p=0.004)$. GOOSS after treatments identified $16(100 \%)$ and $3(33.3 \%)$ patients with a score of 3 in the SPGJ-BEE and EMSP groups, respectively (Table III). Consequently, post-therapeutic GOOSS scores were significantly higher in the SPGJ-BEE group than in the EMSP group $(p<0.001)$. All patients in both groups received chemotherapy after treatments. The mean times $( \pm \mathrm{SD})$ from treatments to the initiation of chemotherapy in the SPGJBEE and EMSP groups were $12.0 \pm 4.5$ and $12.0 \pm 15.0$ days, respectively $(p=0.118)$. 
Table II. Adverse events.

\begin{tabular}{lccc}
\hline Adverse event & $\begin{array}{c}\text { SPGJ-BEE }(\%) \\
(\mathrm{n}=16)\end{array}$ & $\begin{array}{c}\text { EMSP }(\%) \\
(\mathrm{n}=9)\end{array}$ & $p$-Value \\
\hline Bleeding & $0(0.0)$ & $1(11.1)$ & 0.360 \\
Pneumonia & $0(0.0)$ & $1(11.1)$ & 0.360 \\
Abscess or surgical site infection & $1(6.3)$ & $0(0.0)$ & 0.360 \\
Perforation or leakage & $0(0.0)$ & $0(0.0)$ & - \\
Delayed gastric emptying & $0(0.0)$ & $3(11.1)$ & 0.360 \\
Re-obstruction & $0(0.0)$ & $2(22.3)$ & 0.037 \\
Further intervention for re-obstruction & $0(0.0)$ & 0.120 \\
\hline
\end{tabular}

SPGJ-BEE, Stomach-partitioning gastrojejunostomy with Braun enteroenterostomy; EMSP, endoscopic metallic stent placement.

Table III. Clinical courses.

\begin{tabular}{lccc}
\hline Factor & $\begin{array}{c}\text { SPGJ-BEE }(\%) \\
(\mathrm{n}=16)\end{array}$ & $\begin{array}{c}\text { EMSP }(\%) \\
(\mathrm{n}=9)\end{array}$ & $p$-Value \\
\hline Time to a liquid diet (mean days) & $3.4 \pm 2.0$ & $0.9 \pm 0.6$ & $<.001$ \\
Time to a solid diet (mean days) & $4.8 \pm 1.9$ & $2.4 \pm 1.6$ & 0.004 \\
GOOSS scores after treatments (mean) & $0(0.0)$ & $6(66.7)$ & $<0.001$ \\
II & $16(100.0)$ & $3(33.3)$ & - \\
III & $16(100.0)$ & $9(100.0)$ & $0(0.0)$ \\
Chemotherapy after treatments & $0(0.0)$ & $12.0 \pm 15.0$ & 0.118 \\
$\quad$ Yes & $12.0 \pm 4.5$ & \\
No & & \\
Time to the initiation of chemotherapy, mean days &
\end{tabular}

SPGJ-BEE, Stomach-partitioning gastrojejunostomy with Braun enteroenterostomy; EMSP, endoscopic metallic stent placement; GOOSS, gastric outlet obstruction scoring system.

Prognosis. The median survival times of patients in the SPGJBEE and EMSP groups were 414 and 303 days, respectively (Figure 2). No significant differences were observed in survival between the SPGJ-BEE and EMSP groups $(p=0.077)$.

\section{Discussion}

In the present study, we compared clinicopathological characteristics, adverse events, post-therapeutic clinical courses and prognoses between the SPGJ-BEE and EMSP groups in order to assess the clinical impact of SPGJ-BEE on patients with GOO caused by unresectable gastric cancer. To date, a large number of studies have evaluated the clinical benefits of GJ and EMSP for patients with GOO (18-20). However, most of these studies compared clinical outcomes between EMSP and conventional GJ (18-20). To the best of our knowledge, there have been no comparative studies between EMSP and SPGJ plus BEE in patients with GOO caused by unresectable gastric cancer. Consequently, this is the first study to have assessed the clinical impact of SPGJBEE in patients with GOO.
Patients in the SPGJ-BEE group were significantly younger and had better PS than those in the EMSP group in the present study $(p<0.05)$. None of the patients with $\geq P S 2$ underwent SPGJ-BEE. These results may have been influenced by surgery under general anesthesia. Moreover, most patients in the SPGJ-BEE group had tumors located on the lower portion of the stomach. These results indicate the clinical disadvantage and limitation to selecting SPGJ-BEE as an initial treatment for GOO. Accordingly, good PS and tumors located on the lower portion of the stomach are clinical indications for selecting SPGJ-BEE for patients with GOO.

In the present study, a surgical site infection occurred in one out of sixteen patients in the SPGJ-BEE group. None of the patients in the SPGJ-BEE group developed DGE. Ernberg et al. (11) performed a study on 24 patients with GOO who underwent SPGJ or conventional GJ and reported that the incidences of DGE in the SPGJ and conventional GJ groups were $0 \%$ and $42.9 \%$, respectively. These findings indicate the greatest advantage of SPGJ in the surgical approach. Since DGE is directly associated with postoperative quality of life, it is strategically important to 
prevent it in clinical management. Furthermore, the incidences of re-obstruction caused by tumor overgrowth in the EMSP and SPGJ-BEE groups were $33.3 \%$ and $0 \%$, respectively. This result reveals a clinical issue for patients after EMSP.

Kaminishi et al. (9) examined 21 unresectable gastric cancer patients with GOO who underwent SPGJ without BEE or conventional GJ and were the first to report that the intake rates of a regular meal 2 weeks after surgery in the SPGJ and conventional GJ groups were $88 \%$ and $31 \%$, respectively. Furthermore, Kubota et al. (10) examined 25 unresectable cancer patients with GOO who underwent SPGJ without BEE or EMSP and reported that the intake rates of a regular meal 2 weeks after surgery in the SPGJ and EMSP groups were $75 \%$ and $11.1 \%$, respectively. In the present study, patients in the EMSP group had significantly shorter times to a liquid or solid diet than those in the SPGJ-BEE group $(p<0.005)$. However, GOOSS scores 2 weeks after treatments were significantly higher in the SPGJ-BEE group than in the EMSP group $(p<0.001)$. These results indicate that SPGJ-BEE is associated with a better long-term quality of life than EMSP. The maintenance of optimal physical conditions is clinically important for continuous chemotherapy in these patients. Moreover, the early induction and continuation of chemotherapy provides an improved prognosis in patients with unresectable gastric cancer (14). Although survival differences between the two groups were not significantly different in the present study, the median survival time in patients in the SPGJ-BEE group was slightly longer than that in patients in the EMSP group (414 days vs. 303 days). Accordingly, the good long-term quality of life produced by SPGJ-BEE may have an important impact on improved prognoses in patients with GOO caused by unresectable gastric cancer.

There were several limitations to this study. This study was based on retrospective data reviewed in a single institution. Therefore, this non-prospective analysis may have resulted in a selection bias of clinicopathological characteristics between the SPGJ-BEE and EMSP groups. Furthermore, we assessed the clinical impact of SPGJ-BEE on the basis of information obtained from the small patient sample. Consequently, larger validation studies are warranted to strengthen our results.

In conclusion, we herein demonstrated that SPGJ-BEE has impact on improved long-term quality of life and the early induction of subsequent chemotherapy related with a better prognosis in patients with GOO caused by unresectable gastric cancer.

\section{Conflicts of Interest}

The Authors have no conflicts of interest or financial ties to disclose.

\section{References}

1 Katanoda $\mathrm{K}$ and Yako-Suketomo H: Comparison of time trends in stomach cancer incidence (1973-2002) in Asia, from Cancer Incidence in Five Continents, Vols IV-IX. Jpn J Clin Oncol 39: 71-72, 2009.

$2 \mathrm{Li}$ ZX and Kaminishi M: A comparison of gastric cancer between Japan and China. Gastric Cancer 12: 52-53, 2009.

3 Watanabe A, Maehara Y, Okuyama T, Kakeji Y, Korenaga D and Sugimachi K: Gastric carcinoma with pyloric stenosis. Surgery 123: 330-334, 1998.

4 Ye BW, Lee KC, Hsieh YC, Li CP, Chao Y, Hou MC and Lin HC: Self-Expandable Metallic Stent Placement in Malignant Gastric Outlet Obstruction: A Comparison Between 2 Brands of Stents. Medicine (Baltimore) 94: e1208, 2015.

5 Dormann A, Meisner S, Verin N and Wenk Lang A: Self-expanding metal stents for gastroduodenal malignancies: systematic review of their clinical effectiveness. Endoscopy 36: 543-550, 2004.

6 Mimatsu K, Oida T, Kawasaki A, Kano H, Kuboi Y, Aramaki O and Amano S: Laparoscopic-assisted stomach-partitioning gastrojejunostomy for the palliation of gastric outlet obstruction from antral gastric cancer. Surg Laparosc Endosc Percutan Tech 19: e 76-79, 2009.

7 Doberneck RC and Berndt GA: Delayed gastric emptying after palliative gastrojejunostomy for carcinoma of the pancreas. Arch Surg 122: 827-829, 1987.

8 Woods SD and Mitchell GJ: Delayed return of gastric emptying after gastroenterostomy. Br J Surg 76: 145-148, 1989.

9 Kaminishi M, Yamaguchi H, Shimizu N, Nomura S, Yoshikawa A, Hashimoto M, Sakai S and Oohara T: Stomach-partitioning gastrojejunostomy for unresectable gastric carcinoma. Arch Surg 132: 184-187, 1997.

10 Kubota K, Kuroda J, Origuchi N, Kaminishi M, Isayama H, Kawabe T, Omata $M$ and Mafune K: Stomach-partitioning gastrojejunostomy for gastroduodenal outlet obstruction. Arch Surg 142: 607-611, 2007.

11 Ernberg A, Kumagai K, Analatos A, Rouvelas I, Swahn F, Lindblad M, Lundell L, Nilsson M and Tsai JA: The Added Value of Partial Stomach-partitioning to a Conventional Gastrojejunostomy in the Treatment of Gastric Outlet Obstruction. J Gastrointest Surg 19: 1029-1035, 2015.

$12 \mathrm{Xu} \mathrm{B}$, Meng H, Qian M, Gu H, Zhou B and Song Z: Braun enteroenterostomy during pancreaticoduodenectomy decreases postoperative delayed gastric emptying. Am J Surg 209: 10361042, 2015.

$13 \mathrm{Xu}$ B, Zhu YH, Qian MP, Shen RR, Zheng WY and Zhang YW: Braun Enteroenterostomy Following Pancreaticoduodenectomy: A Systematic Review and Meta-Analysis. Medicine (Baltimore) 94: e1254, 2015.

14 Okumura Y, Yamashita H, Aikou S, Yagi K, Yamagata Y, Nishida M, Mori K, Nomura S, Kitayama J, Watanabe T and Seto Y: Palliative distal gastrectomy offers no survival benefit over gastrojejunostomy for gastric cancer with outlet obstruction: retrospective analysis of an 11-year experience. World J Surg Oncol 12: 364, 2014.

15 Edge SB, Byrd DR, Compton CC, Fritz AG, Greene FL and Trotti A: AJCC Cancer Staging Manual, ed 7. Springer, New York, 2010.

16 Adler DG and Baron TH: Endoscopic palliation of malignant gastric outlet obstruction using self-expanding metal stents: experience in 36 patients. Am J Gastroenterol 97: 72-78, 2002. 
17 Wente MN, Bassi C, Dervenis C, Fingerhut A, Gouma DJ, Izbicki JR, Neoptolemos JP, Padbury RT, Sarr MG, Traverso LW, Yeo CJ and Büchler MW: Delayed gastric emptying (DGE) after pancreatic surgery: a suggested definition by the International Study Group of Pancreatic Surgery (ISGPS). Surgery 142: 761-768, 2007.

18 Jeurnink SM, Steyerberg EW, van Hooft JE, van Eijck CH, Schwartz MP, Vleggaar FP, Kuipers EJ and Siersema PD; Dutch SUSTENT Study Group: Surgical gastrojejunostomy or endoscopic stent placement for the palliation of malignant gastric outlet obstruction (SUSTENT study): A multicenter randomized trial. Gastrointest Endosc 71: 490-499, 2010.

19 Khashab M, Alawad AS, Shin EJ, Kim K, Bourdel N, Singh VK, Lennon AM, Hutfless S, Sharaiha RZ, Amateau S, Okolo PI,
Makary MA, Wolfgang C, Canto MI and Kalloo AN: Enteral stenting versus gastrojejunostomy for palliation of malignant gastric outlet obstruction. Surg Endosc 27: 2068-2075, 2013.

20 No JH, Kim SW, Lim CH, Kim JS, Cho YK, Park JM, Lee IS, Choi MG and Choi KY: Long-term outcome of palliative therapy for gastric outlet obstruction caused by unresectable gastric cancer in patients with good performance status: Endoscopic stenting versus surgery. Gastrointest Endosc 78: 55-62, 2013.

Received July 30, 2016

Revised August 19, 2016

Accepted August 23, 2016 